\title{
Socioeconomic and lifestyle factors associated with mental health problems among Mongolian elementary school children
}

\author{
Ai Aoki ${ }^{1,2}$ (1) $\cdot$ Ganchimeg Togoobaatar ${ }^{3}(0) \cdot$ Anudari Tseveenjav $^{4,5} \cdot$ Naranbaatar Nyam $^{4} \cdot$ Khishigsuren Zuunnast $^{6}$. \\ Gundegmaa Lkhagvasuren $^{7}$ (1) $\cdot$ Bat-Erdene Shagdar $^{7}$ (1) $\cdot$ Rintaro Mori $^{8}$ (D) $\cdot$ Akihito Kikuchi $^{9}$ (D) $\cdot$ Hideaki Soya $^{9,10}$ (1) \\ Kiyoto Kasai ${ }^{2} \cdot$ Kenji Takehara' $^{1}$
}

Received: 28 June 2021 / Accepted: 22 September 2021 / Published online: 30 September 2021

(c) Springer-Verlag GmbH Germany, part of Springer Nature 2021

\begin{abstract}
Purpose Lifestyle factors of children and adolescents' mental health problems are an emerging health issue in low- and middle-income countries (LMICs). However, there is a lack of studies on lifestyle factors in LMICs. This study examined the socioeconomic and lifestyle factors associated with mental health problems among school-age children in Mongolia.

Methods A population-based cross-sectional survey was conducted among 4th-year students at public elementary schools in one district in Ulaanbaatar. The Strengths and Difficulties Questionnaire (SDQ) and a self-administrated socioeconomic and lifestyle questionnaire were completed by participants' guardians. A multivariate logistic regression analysis was performed. Results Of the 2301 children surveyed, 1694 without missing responses were included in the analysis. The multivariate logistic regression analysis showed that male gender [adjusted odds ratio (AOR) 1.64 (1.29-2.10)], low maternal education [AOR 1.89 (1.16-3.05)], short sleep [AOR 1.41 (1.10-1.80)], no physical activity [AOR 1.31 (1.03-1.67)], and long screen time (AOR $1.53(1.20-1.94)$ ) were associated with high risk of mental health problems. Low maternal education, low household income, no physical activity habit, and long screen time were associated with internalising problems. Meanwhile, male gender, low maternal education, and long screen time were associated with externalising problems.

Conclusion The results are consistent with previous studies in high-income countries, indicating that there are globally common socioeconomic and lifestyle risk factors. The findings of this study may help develop a targeted preventive intervention for high-risk groups, such as socioeconomically disadvantaged groups, as well as a universal preventive intervention to foster a healthy lifestyle in Mongolia.
\end{abstract}

Keywords Child and adolescent mental health $\cdot$ Developing country $\cdot$ Socioeconomic factor $\cdot$ Physical activity $\cdot$ Screen time

Ai Aoki

aaoki-tky@umin.ac.jp

1 Department of Health Policy, National Center for Child Health and Development, 2-10-1 Okura, Setagaya, Tokyo, Japan

2 Department of Neuropsychiatry, Graduate School of Medicine, University of Tokyo, Tokyo, Japan

3 Faculty of Medicine, Global Health Nursing, University of Tsukuba, Tsukuba, Ibaraki, Japan

4 School of Nursing, Mongolian National University of Medical Sciences, Ulaanbaatar, Mongolia

5 Global Leadership University, Ulaanbaatar, Mongolia
6 Department of Mental Health, School of Medicine, Mongolian National University of Medical Sciences, Ulaanbaatar, Mongolia

7 Mongolian National Institute of Physical Education, Ulaanbaatar, Mongolia

8 Graduate School of Medicine, Kyoto University, Sakyoku, Kyoto, Japan

9 Advanced Research Initiative for Human High Performance, Faculty of Health and Sport Sciences, University of Tsukuba, Ibaraki, Japan

10 Laboratory of Exercise Biochemistry and Neuroendocrinology, Faculty of Health and Sport Sciences, University of Tsukuba, Ibaraki, Japan 


\section{Introduction}

Children's and adolescents' mental health is an important health issue. Globally, about $10-20 \%$ of children and adolescents experience some form of mental health problem [1]. In low-middle-income countries (LMICs), where about $90 \%$ of the child population in the world reside [2], it has been estimated that $13.2-20.1 \%$ of years lived with disability among children under 20 years of age was attributable to mental disorders and $0.4-1.1 \%$ to substance use disorder in 2019 [3]. As childhood mental health problems can lead to lasting social and professional impairment, the prevention, detection, and treatment of mental health problems in children and adolescents are important [4].

In addition to basic socioeconomic risk factors [5], the influence of a modern lifestyle on children's mental health is gaining attention. With lifestyles becoming more modernised, children are spending less time on physical activities and more time on sedentary activities, especially those involving digital devices (screen time). A day consists of sleep time, sedentary time, and various intensity physical activities. Moreover, due to the lifestyle changes brought about by the COVID-19 pandemic, more attention is being paid to the influence of modernised lifestyles on children's and adolescents' mental health $[6,7]$. Systematic reviews have shown that physical inactivity has association with depression and anxiety among children and adolescents [8], and that screen time is associated with depression among children and adolescents $[9,10]$. Some studies demonstrated that long screen time causes attention problems among children $[11,12]$. One meta-analysis reported that sleep loss is associated with depression [13]. According to another systematic review, physical inactivity, long sedentary time and short sleep together pose higher risk on children's and adolescents' depression [14]. Considering the various risks as a result of lifestyle behaviours, the World Health Organisation recommends increasing physical activities, reducing sedentary time-especially screen time-among children, and initiating national plan to promote these healthy habits in each country [15]. Canada and Australia developed 24-h movement guidelines and recommend $60 \mathrm{~min}$ or more of moderate to vigorous physical activity per day, no more than 120 min of leisure screen time per day, and $9-11 \mathrm{~h}$ of sleep for children aged $5-13$ years [16, 17]. However, most countries that have implemented national policies to address lifestyle changes among children are high-income countries (HICs).

In LMICs, where epidemiological transition is occurring, the impact of children's and adolescents' mental health problems including psychological developmental problems is increasing. However, it is known that child and adolescent mental health problems have been severely underserved in most LMICs [18]. Untreated mental health problems among children and youth in LMICs have longterm effects such as poor academic attainment, lost productivity, and chronic disability in adulthood and ultimately hinder development at the national level [18]. Understanding the risk factors for mental health and behavioural problems is highly beneficial as it helps develop and implement preventive interventions [19, 20]. However, even basic socioeconomic risk factors have not been fully investigated in LMICs. Several studies conducted in LMICs have suggested an association between low socioeconomic status (e.g. low maternal education and one-parent family) and children's mental health and behavioural problems [21-25]. Regarding lifestyle factors, analyses of the Global school-based Student Health Survey revealed that long sedentary time increases the odds of depressive and anxiety symptoms among adolescents from LMICs [26, 27]. Nevertheless, very few studies have investigated the association between lifestyle factors and mental health using a valid assessment tool $[28,29]$. To promote child and adolescent mental health in LMICs, there is a need to elucidate whether there exist similar basic risk factors and similar preventive strategies are applicable in LMICs compared to HICs. Considering the obvious lack of evidence, more studies of basic child and adolescent mental health risk factors are needed in LMICs.

There is a paucity of studies pertaining to mental health and its associated factors among children and adolescents in Mongolia. Thus, this study aimed to identify the socioeconomic and lifestyle factors associated with children's mental health problems, using data from a population-based study on school-age children in the capital city of Mongolia. Children's mental health was assessed using a validated mental health screening tool, and their socioeconomic factors, physical activity time, screen time, and sleep duration were analysed to determine their association with children's mental health. In addition to contributing to the Mongolian health policy, this study sought to contribute to the literature on child and adolescent mental health in LMICs-particularly countries where the epidemiological transition is occurring-by accumulating evidence.

\section{Methods}

\section{Study design}

This is a cross-sectional analysis. We analysed a part of the data from a randomised controlled trial to examine the effectiveness of exercise intervention for academic achievement, cognitive function, and physical health among children in Mongolia, which was conducted by our research team in 
2018. Details of the study methodology and results are provided elsewhere [30, 31].

\section{Study setting}

The original randomised controlled trial (RCT) was conducted in the Sukhbaatar district of Ulaanbaatar, the capital city of Mongolia. Mongolia is a lower-middle-income and former communist country in Western Pacific Asia with a population of approximately $3,170,000$ people [32]. The country has already developed a primary healthcare and a social insurance system, and the health standards of Mongolian people are above the average levels compared to the health standards of citizens of countries with a similar economic level [33]. Mental health needs are considered to be high according to previous surveys that reported that a considerable proportion of children and young people have mental health concerns [34-36]. To date, only one risk factor analysis has been performed in Mongolia, and it revealed a strong association between family factors (e.g. maternal depression and children's behavioural problem) and mental health among young children in a rural area [35].

The capital city, Ulaanbaatar, is the main cultural, industrial and administrative centre of the country, where almost half of the country's population resides. It has nine districts with no distinct differences in administration, socioeconomic conditions, health, and cultural factors among inhabitants. The population of the Sukhbaatar district represents roughly $10 \%$ of the population of Ulaanbaatar [37].

\section{Study population}

Participants in this study were the participants of a baseline survey of the original RCT. The original RCT recruited all fourth-year students from 10 of the 11 public elementary schools in the district (one school participated in the pilot phase) and examined the effectiveness of a short exercise programme on their achievement at the national examination in a 10-month follow up. Inclusion criteria were: (1) attendance at one of the 10 schools that participated in the study; (2) written consent from parents/guardians; and (3) age-appropriate Mongolian literacy. Exclusion criteria were (1) presence of comorbidities or contraindications prohibiting participation in an exercise programme; and (2) enrolment in a special needs programme. In Mongolia, inclusive education for children with special needs is provided in mainstream schools. Children with mild disabilities who are diagnosed by doctors are enrolled in the regular programme. Those who have severe disabilities are enrolled in special schools. However, dropout rate among children with disabilities is high due to inadequate school resource [38].

This study was approved by the ethical committee at National Center for Child Health and Development, Tokyo,
Japan (Number 1541) and the Mongolian National Institute for Physical Education, Ulaanbaatar, Mongolia (Number 238).

Written consent was obtained from the primary guardians prior to the original RCT. Researchers encouraged guardians to explain the study to their children, but informed assent was not obtained from the children.

\section{Measures}

Demographic, socioeconomic, and lifestyle factors were collected using a self-administered questionnaire distributed to the participants' guardians. Mental health was measured using the parental version of the SDQ [39-42].

\section{Socioeconomic factors}

The socioeconomic factors included living area, family structure, maternal education, and household income. Living area was a binary variable: (1) urban area and (2) nonurban area (i.e., infrastructure is less developed), according to the administrative category of the school location. Family structure had two categories: (1) two-parent family, and (2) one-parent/no-parent family. Maternal education was measured as the highest level that the mother achieved and converted into a binary variable: (1) low education (lower secondary education or less, 9 years and less) and (2) middle to high education (upper secondary education and more, 10 years and more). Household income was categorised into eight groups and converted into binary variable: (1) low income and (2) middle to high income. Low income was determined by the threshold of 700,000 Mongolian Tugrik (MNT) per month, which was equal to about USD 280 in 2018, when this study was conducted [37]. According to the Mongolian national statistics, the proportion of households whose household income was below 700,000 MNT was 23.9\% in Ulaanbaatar in 2019 and closest to the poverty headcount (25.9\%) in Ulaanbaatar in 2018 [37, 43].

\section{Lifestyle factors}

Lifestyle factors were selected according to 24-h movement guidelines from Canada and Australia [16, 17] and sleep, physical activity, and screen time were included. Sleep was measured by sleep hours and converted into two categories by the guideline recommendation [16, 17]: (1) short sleep (less than nine hours per day), and (2) recommended sleep and more (nine hours per day and more). Physical activity was measured by the frequency of children's moderate to vigorous physical activity (physical activity that causes the child to sweat and breathe harder) besides physical education classes. Frequency of moderate to vigorous physical activity was grouped into five categories and 
converted to two categories: (1) not engaging in physical activity (less than once per week), and (2) engaging in physical activity (once or more per week). The reason for measuring the frequency of moderate to vigorous physical activity per week to indicate exercise habit was based on the general trend of Mongolian children being physically inactive due to urbanisation, air pollution, and severe winter coldness. Global School-based Student Health Survey in 2013 demonstrated that $25.2 \%$ of school children were engaged in the recommended level of physical activity, which was one hour of moderate physical activity per day [36, 44].

Screen time was grouped into five categories and converted to two categories according to the guideline recommendation [16, 17]: (1) recommended screen time (less than $2 \mathrm{~h}$ per day), and (2) long screen time ( $2 \mathrm{~h}$ and more per day).

\section{Mental health}

The SDQ has been widely used in international studies to screen a range of behavioural problems among children and adolescents aged 4-17 years. The SDQ consists of 25 simple questions on strengths and difficulties across four difficulty subscales: (1) emotion, (2) conduct, (3) hyperactivity, and (4) peer problems, as well as one strength subscale. Summing the four difficulty subscale scores yields the total difficulties score; summing emotion and peer problems subscales yields the internalising score; and summing conduct and hyperactivity subscales yields the externalising score [45]. Using these scores, the levels of risk were categorised as abnormal, borderline, and normal by the normative banding that categorizes children with the highest $10 \%$ score range as abnormal and the second highest $10 \%$ as borderline following the original method [41]. The SDQ has already been validated in Mongolia by a previous study that compared a community population (the same study population as this study) and a clinical population comprising patients at a child and adolescent psychiatric outpatient service at the National Mental Health Center [42]. In this study, mental health problems were assessed using the SDQ total difficulties score, internalising score, and externalising score. This study adopted a total difficulties cut-off score for borderline, which was recommended for the screening of community children in the previous study [42]. The cut-off scores for internalising and externalising behaviours were determined by the same level of normative banding-categorising participants above the 80th percentile as high risk. Using these cut-off scores, the total difficulties score, internalising score, and externalising score were converted into a binary variable: (1) high risk (total difficulties score of 17 or higher, internalising/externalising score above 80th percentile); and (2) low risk (total difficulties score of 16 or lower, internalising/externalising score below 80th percentile). Each subscale was not used due to their poor fit to the five-factor model in the Mongolian context [42].

\section{Statistical analysis}

\section{Descriptive analysis}

The participants' socioeconomic variables were descriptively analysed and compared between participants with and without missing values among the explanatory variables and the SDQ, to address any selection bias. For age, a $t$ test was performed. For variables other than age, a chi-squared test was performed. Among participants without missing values, explanatory variables were compared between high-risk and low-risk participants. All the explanatory variables were categorical variables, and a chi-squared test was performed.

The SDQ total difficulties score, internalising score, and externalising scores were descriptively analysed.

\section{Normative banding of internalising and externalising scores}

Normative distribution tables for the internalising and externalising score were created. The cut-off scores were set around the 80 th percentile level so that the highest $20 \%$ was categorised as high-risk, which is equivalent to the cut-off of the total difficulties score in Mongolia.

\section{Logistic regression analysis}

The explanatory variables included in the logistic regression analysis comprised gender, living area, family structure, maternal education, household income, sleep, physical activity, and screen time. Reference categories were as follows: gender: female; living area: urban area; family structure: two-parent family; maternal education: middle to high education; household income: middle to high income; sleep: recommended sleep; physical activity: engaging in physical activity; and screen time: recommended screen time. As the participants were students of the same year in elementary school and because their age was considered to be virtually the same, age was not included as an explanatory variable. The dependent variable was high-risk of mental health problems based on the total difficulties score, internalising score, and externalising score. All the explanatory variables were hypothesised to be associated with the dependent variable. Thus, a forced entry method was used. Multicollinearity was examined by variance inflation factor.

First, the association between mental health problems and each explanatory variable was analysed using a univariate logistic regression analysis, after which the crude odds ratio was calculated. Second, the association between mental health problems and all the explanatory 
variables was analysed using a multivariate logistic regression analysis, after which the adjusted odds ratio was calculated. Statistical significance threshold was set at $p=0.05$.

Missing values were not complemented. Participants without missing values in all the explanatory and dependent variables were included in the analysis.

All analyses were conducted using R software, version 3.6.

\section{Results}

\section{Participants}

A total of 2309 children were enrolled in the 10 schools participating in the original RCT study. Of these, 2301 children provided consent to participate. None of them met the exclusion criteria. Of the 2301 children, 1694 children $(82.8 \%)$ did not have any missing values among the SDQ and explanatory variables, hence were included in the logistic regression analysis. One hundred and fifty-one (10.9\%) participants did not answer any item of the self-administered questionnaire or SDQ.

\section{Description of participants}

The mean age of the participants was 9.7 (SD: 0.4), and 1,051 (51.3\%) participants were male. Other socioeconomic variables are presented in Table 1.

\section{Comparison of participants with and without missing values}

Age and gender did not differ between 1694 participants without missing values (73.6\%, analysed participants) and 607 participants with missing values $(26.4 \%$, not analysed participants). Socioeconomic variables, such as living area, family structure, maternal education, and household income, differed between the two groups. The group with missing values had a larger proportion of individuals with low socioeconomic status (Table 1). Thus, a greater number of participants from low socioeconomic groups were excluded from the analysis.
Table 1 Demographic and socioeconomic characteristics of participants and comparison of participants without missing values and with missing values

\begin{tabular}{|c|c|c|c|c|c|c|c|}
\hline & \multicolumn{2}{|c|}{$\begin{array}{l}\text { All participants } \\
n=2301\end{array}$} & \multicolumn{2}{|c|}{$\begin{array}{l}\text { Without missing value } \\
\text { (analysed participants) } \\
n=1694\end{array}$} & \multicolumn{2}{|c|}{$\begin{array}{l}\text { With missing value } \\
\text { (not analysed partici- } \\
\text { pants) } \\
n=607\end{array}$} & \multirow[t]{2}{*}{$P$ value $^{\mathrm{a}}$} \\
\hline & $n$ & $\%$ & $n$ & $\%$ & $n$ & $\%$ & \\
\hline Age (mean/SD) & 9.7 & 0.4 & 9.7 & 0.4 & 9.8 & 0.5 & 0.13 \\
\hline Gender & & & & & & & 0.36 \\
\hline Male & 1051 & $51.3 \%$ & 861 & $50.8 \%$ & 190 & $53.7 \%$ & \\
\hline Female & 997 & $48.7 \%$ & 833 & $49.2 \%$ & 164 & $46.3 \%$ & \\
\hline Missing & 253 & & 0 & & 253 & & \\
\hline Living area & & & & & & & $<0.001$ \\
\hline Urban & 1415 & $61.5 \%$ & 1085 & $64.0 \%$ & 330 & $54.4 \%$ & \\
\hline Not-urban & 886 & $38.5 \%$ & 609 & $36.0 \%$ & 277 & $45.6 \%$ & \\
\hline Missing & 0 & & 0 & & 0 & & \\
\hline Family structure & & & & & & & $<0.001$ \\
\hline Two-parent & 1644 & $80.3 \%$ & 1410 & $83.2 \%$ & 234 & $66.1 \%$ & \\
\hline One/no-parent & 404 & $19.7 \%$ & 284 & $16.8 \%$ & 120 & $33.9 \%$ & \\
\hline Missing & 253 & & 0 & & 253 & & \\
\hline Maternal education & & & & & & & $<0.001$ \\
\hline Middle-high & 1786 & $94.0 \%$ & 1605 & $94.7 \%$ & 181 & $88.3 \%$ & \\
\hline Low & 113 & $6.0 \%$ & 89 & $5.3 \%$ & 24 & $11.7 \%$ & \\
\hline Missing & 402 & & 0 & & 402 & & \\
\hline Household income & & & & & & & $<0.001$ \\
\hline Middle-high & 1319 & $65.1 \%$ & 1161 & $68.5 \%$ & 158 & $47.4 \%$ & \\
\hline Low & 708 & $34.9 \%$ & 533 & $31.5 \%$ & 175 & $52.6 \%$ & \\
\hline Missing & 274 & & 0 & & 274 & & \\
\hline
\end{tabular}

${ }^{\mathrm{a}}$ For comparison between participants without and with missing values, a $t$ test was performed for a continuous variable (age), and a chi-squared test was performed for categorical variables (variables other than age) 
The mean total difficulties score on the SDQ was 12.9 (SD: 4.9) among participants without missing values and 13.3 (SD: 4.8) among participants with missing values, although there was no statistically significant difference.

\section{Results of the SDQ}

Among all participants, the mean total difficulties score was 12.9 (SD: 4.8). The mean internalising score was 6.7 (SD: 2.9), and the mean externalising score was 6.2 (SD: 3.2).

Using normative distribution and its 80th-percentile level, a cut-off score of 10 was set for the internalising score 9 for externalising score. Based on these cut-off scores, of the 2046 participants who answered the SDQ, 349 (17.1\%) participants were classified as high risk for internalising, and $461(22.5 \%)$ participants were classified as high risk for externalising. Normative distribution is presented as Supplementary Table 1.

Of the 440 children who had an SDQ total difficulties score above the high-risk cut-off score, 245 (55.7\%) were classified as high risk for internalising problems and 308 (70.0\%) for externalising problems. The distribution of each score is presented in Fig. 1.

\section{Multicollinearity}

The variant inflation factor was $<10$ between all the explanatory variables. The highest was 1.11 between living area and household income.

\section{Logistic regression analysis}

\section{Total difficulties score}

A univariate logistic regression analysis and a multivariate logistic regression analysis were performed, using total difficulties score as an indicator of the presence of a mental health problem (Table 2). The crude odds ratio for male gender was 1.59 (95\%CI 1.26-2.02), 1.39 for nonurban (95\% CI 1.10-1.77), 2.07 for low maternal education (95\% CI 1.3-3.22), 1.56 for low household income (95\% CI 1.23-1.99), 1.37 for short sleep (95\% CI 1.08-1.74), 1.32 for not engaging in physical activity (95\% CI 1.05-1.67), and 1.57 for long screen time (95\%CI 1.24-1.99); these were statistically significant in the univariate analysis. Among these estimates, male gender, low maternal education, short sleep, not engaging in physical activity, and long screen time remained significant in the multivariate logistic regression analysis. The adjusted odds ratio for male gender was 1.64 (95\%CI 1.29-2.10), 1.89 for low maternal education (95\% CI 1.16-3.05), 1.41 for short sleep (95\% CI $1.10-1.80), 1.31$ for not engaging in physical activity (95\% CI 1.03-1.67), and 1.53 for long screen time (95\% CI $1.20-1.94)$.

\section{Internalising score}

A univariate logistic regression analysis and a multivariate logistic regression analysis were performed using internalising score as an indicator of the presence of a mental health problem (Table 3 ). The crude odds ratio for low maternal education was 2.29 (95\%CI 1.40-3.65), 1.91 for low household income (95\%CI 1.46-2.49), 1.44 for not engaging in physical activity (95\% CI 1.11-1.87), and 1.33 for long screen time (95\%CI 1.03-1.73), all of which were statistically significant in the univariate and multivariate analyses. The adjusted odds ratio for low maternal education was 1.82 (95\%CI 1.09-2.98), 1.72 for low household income (95\% CI 1.27-2.31), 1.34 for not engaging in physical activity (95\%CI $1.03-1.75)$, and 1.37 for long screen time (95\%CI 1.05-1.79).
Total difficulties score

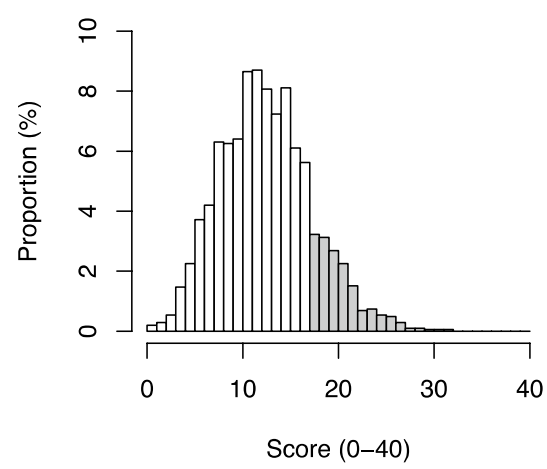

Internalising score

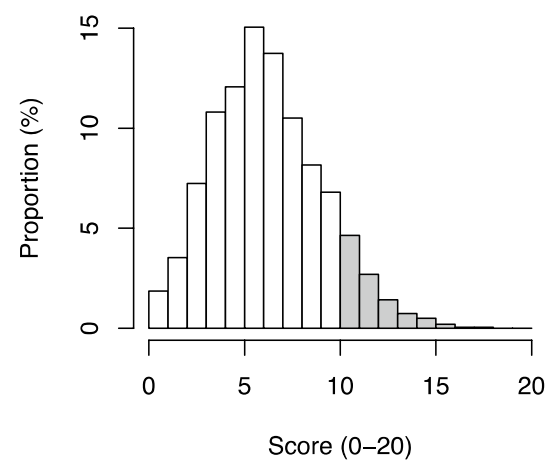

Externalising score



Fig. 1 Distribution of SDQ total difficulties score, internalising and externalising score. Gray highlight indicates high-risk population. The cutoff score of 17 was used for total difficulties score, 10 for internalising score, and 9 for externalising score. 
Table 2 Difference between high-risk and low-risk population defined by total difficulties score with crude and adjusted odds ratios of each explanatory variable

\begin{tabular}{|c|c|c|c|c|c|c|c|}
\hline & \multirow{2}{*}{$\begin{array}{l}\text { Low risk } \\
N(\%)\end{array}$} & \multirow{2}{*}{$\begin{array}{l}\text { High risk } \\
N(\%)\end{array}$} & \multirow{2}{*}{$\begin{array}{l}\text { Chi-square } \\
P \text { value }\end{array}$} & \multicolumn{4}{|l|}{ Odds ratio } \\
\hline & & & & Crude $(95 \% \mathrm{CI})$ & $P$ value & Adjusted $(95 \% \mathrm{CI})$ & $P$ value \\
\hline Gender & & & $<0.001$ & & & & \\
\hline Female & $688(51.6 \%)$ & $145(40.2 \%)$ & & 1 & & 1 & \\
\hline Male & $645(48.4 \%)$ & $216(59.8 \%)$ & & $1.59(1.26-2.02)$ & $<0.001$ & $1.64(1.29-2.10)$ & $<0.001$ \\
\hline Living area & & & 0.01 & & & & \\
\hline Urban & $876(65.7 \%)$ & $209(57.9 \%)$ & & 1 & & 1 & \\
\hline Non-urban & $457(34.3 \%)$ & $152(42.1 \%)$ & & $1.39(1.10-1.77)$ & 0.01 & $1.25(0.96-1.61)$ & 0.09 \\
\hline Family structure & & & 0.04 & & & & \\
\hline Two-parent & $1123(84.2 \%)$ & $287(79.5 \%)$ & & 1 & & 1 & \\
\hline One-parent/no-parent & $210(15.8 \%)$ & $74(20.5 \%)$ & & $1.38(1.02-1.84)$ & 0.03 & $1.29(0.94-1.75)$ & 0.10 \\
\hline Mother's education & & & $<0.01$ & & & & \\
\hline Middle to high & $1275(95.6 \%)$ & $330(91.4 \%)$ & & 1 & & 1 & \\
\hline Low & $58(4.4 \%)$ & $31(8.6 \%)$ & & $2.07(1.30-3.22)$ & $<0.01$ & $1.89(1.16-3.05)$ & 0.01 \\
\hline Household income & & & $<0.001$ & & & & \\
\hline Middle to high & $942(70.7 \%)$ & $219(60.7 \%)$ & & 1 & & 1 & \\
\hline Low & $391(29.3 \%)$ & $142(39.3 \%)$ & & $1.56(1.23-1.99)$ & $<0.001$ & $1.31(0.99-1.72)$ & 0.06 \\
\hline Sleep & & & 0.01 & & & & \\
\hline Recommended sleep and more & $888(66.6 \%)$ & $214(59.3 \%)$ & & 1 & & 1 & \\
\hline Short sleep & $445(33.4 \%)$ & $147(40.7 \%)$ & & $1.37(1.08-1.74)$ & 0.01 & $1.41(1.10-1.80)$ & 0.01 \\
\hline Physical activity & & & 0.02 & & & & \\
\hline Engaging in physical activity & $761(57.1 \%)$ & $181(50.1 \%)$ & & 1 & & 1 & \\
\hline Not engaging in physical activity & $572(42.9 \%)$ & $180(49.9 \%)$ & & $1.32(1.05-1.67)$ & 0.02 & $1.31(1.03-1.67)$ & 0.03 \\
\hline Screen time & & & $<0.001$ & & & & \\
\hline Recommended screen time & $781(58.6 \%)$ & $171(47.4 \%)$ & & 1 & & 1 & \\
\hline Long screen time & $552(41.4 \%)$ & $190(52.6 \%)$ & & $1.57(1.24-1.99)$ & $<0.001$ & $1.53(1.20-1.94)$ & $<0.001$ \\
\hline
\end{tabular}

\section{Externalising score}

A univariate logistic regression analysis and a multivariate logistic regression analysis were performed using externalising score as an indicator of the presence of a mental health problem (Table 3). The crude odds ratios for male gender was 1.78 (95\% CI 1.41-2.25), 1.38 for non-urban area (95\% CI 1.09-1.74), 1.9 for low maternal education (95\% CI 1.19-2.95), 1.41 for low household income (95\% CI 1.11-1.79), 1.71 for long screen time (95\% CI 1.36-2.15), all of which were statistically significant in the univariate analysis. Among these, male gender, low maternal education, and long screen time remained significant in the multivariate logistic regression analysis. The adjusted odds ratio for male gender was 1.79 (95\%CI 1.41-2.27), 1.78 for low maternal education (95\% CI 1.09-2.86), and 1.65 for long screen time (95\% CI 1.30-2.08).

\section{Discussion}

\section{Summary of the results}

Among Mongolian elementary school children, male gender, low maternal education, short sleep, not engaging in physical activity, and long screen time were significant risk factors for general mental health problems. For internalising problems, low maternal education, low household income, not engaging in physical activity, long screen time were significant, whereas male gender, low maternal education, and long screen time were significant for externalising problems. 


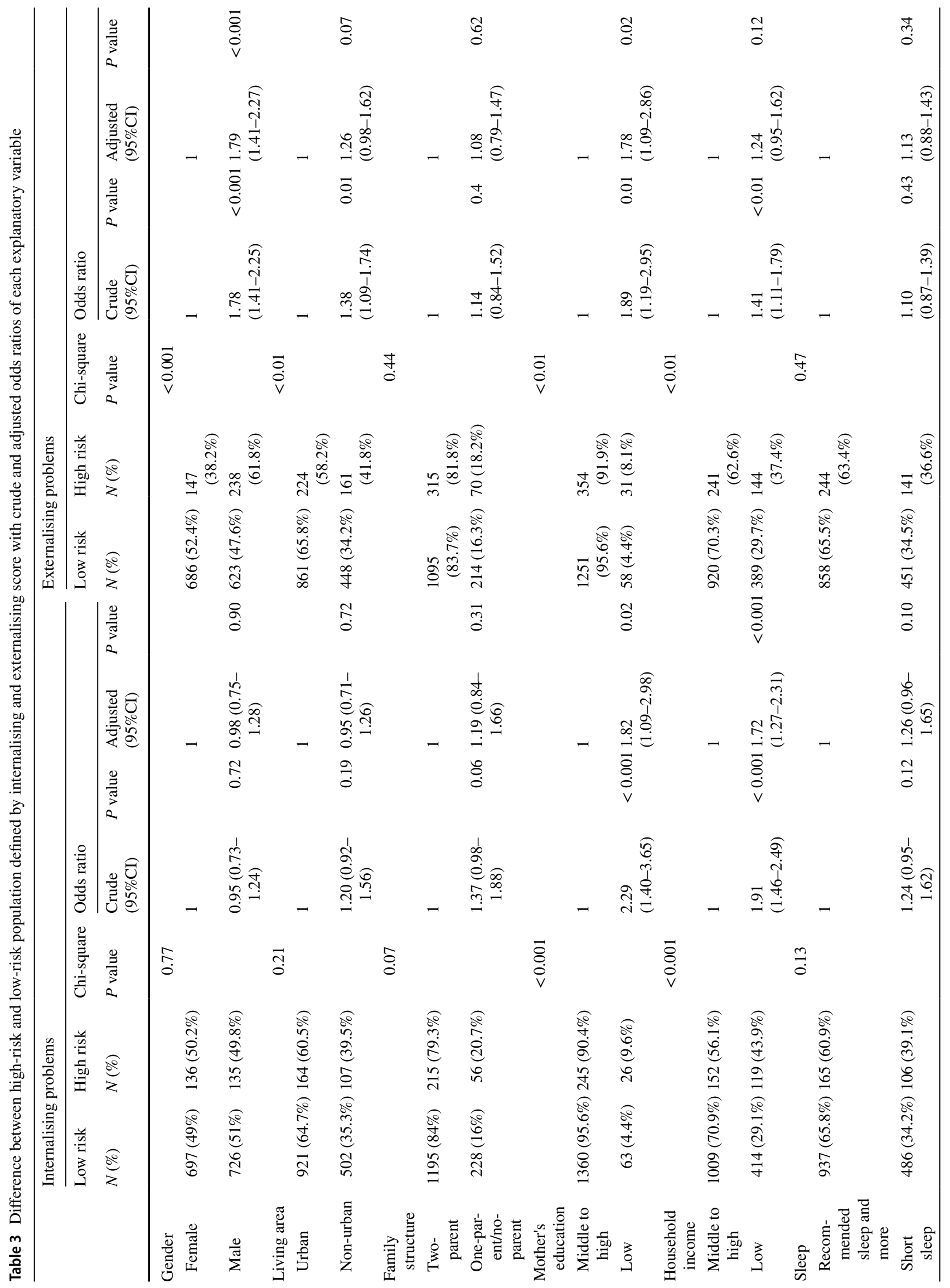




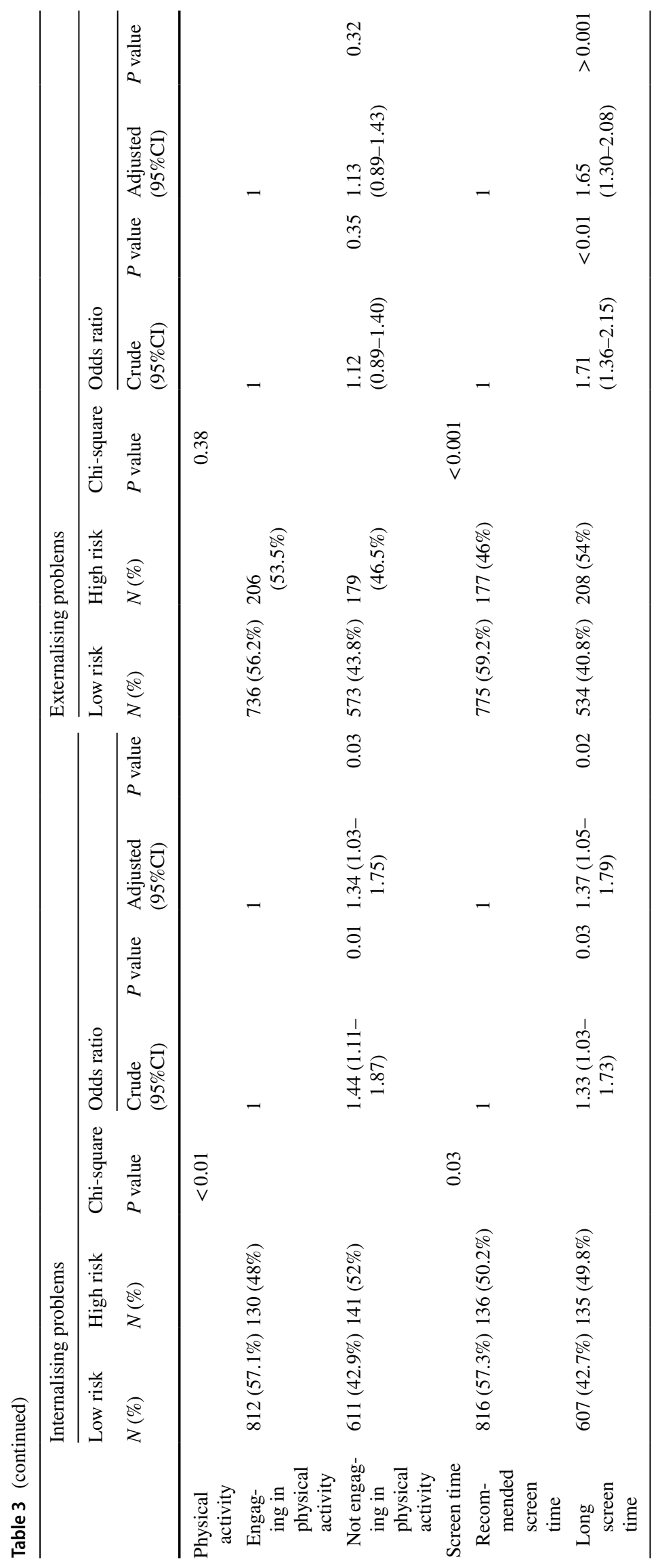




\section{Comparison of the results of general mental health problems with previous studies and extrapolability of the results}

\section{Gender}

The results showed that males are at a higher risk for general mental health problems and externalising problems but not for internalising problems. This coincides with the fact that the prevalence of some childhood mental disorders is higher among males [46-48].

\section{Socioeconomic factors}

Lower maternal education was strongly associated with general mental health problems. Other socioeconomic factors were not consistently associated with mental health problems at a statistically significant level, but the adjusted odds ratios for all factors were around 1 and higher. With larger sample size, other socioeconomic factors might also demonstrate significant association with general mental health problems. This corresponds to previous results about the association between low socioeconomic status and mental health in high-income countries [5]. It has been known that maternal education is an important social determinant of children's health, particularly in LMICs [49]. A previous study in Mongolia also reported an association between subscales of the SDQ and lower maternal education [22].

\section{Lifestyle factors}

This study demonstrated the general association between short sleep, not engaging in physical activity, long screen time and general mental health problems. Although the evidence is stronger for the association between these lifestyle factors and specific mental health symptoms such as depression and anxiety [8-10, 13], some previous studies demonstrated an association between lifestyle factors and general mental health problem [50-52]. This study suggested that multiple lifestyle factors contributed to poorer mental health, which imply that the needs of fostering healthy lifestyle among children are applicable to greater population.

\section{Comparison of the results of internalising problems and externalising problems with previous studies}

Internalising problems refer to depressive or anxiety symptoms and externalising problems refer to hyperactive, inattention or conduct symptoms. The association between physical inactivity, long screen time and internalising problems is consistent with the previous studies mainly conducted in high-income countries [8-10]. Our results demonstrated the association between long screen time and externalising problems which is consistent with emerging evidence on the association between long screen time and inattention problems $[11,12]$. Gender, household income and physical activity demonstrated different impact on internalising and externalising problems. Gender reflects epidemiological difference between boys and girls. The prevalence of some childhood mental disorders which frequently exhibit externalising problems - such as attention deficit hyperactivity disorder, and conduct disorder-is higher among males [46, 47]. Low household income demonstrated significantly higher odds of internalising problems and non-significant higher odds of externalising problems. However, as the lower limit of $95 \%$ confidential interval was close to 1 for externalising problems, the difference might disappear when it is examined with larger sample size. Not engaging in physical activity posed higher odds only for internalising problems. This is consistent with the existing evidence that physical inactivity is a risk factor for depressive or anxiety symptoms, thus this might be a true difference between internalising and externalising problems.

\section{Implications for practice}

Our results indicate that children's lifestyle needs more attention in LMICs. As WHO recommends [15], our results suggests that national plans to promote healthy lifestyle among children is beneficial for children's mental health in LMICs.

In LMICs, where resources are limited, a universal intervention utilising existing resources is more feasible than targeted intervention employing new resources. Regarding lifestyle factors, there have been many studies investigated the effects of school-based and home-based intervention on physical activity and screen time in HICs [53, 54]. As physical activity, screen time and sleep are interrelated, interventions often targeted multiple factors [54, 55]. School might be a most feasible place to deliver intervention to children in LMICs but children are under strong influence of parents [53, 54]. Thus, interventions combining school-based activities and education and support to parents are considered to be effective [55]. For example, school-based physical activity intervention (e.g. increasing the number of physical education classes, improving the quality of physical education classes) and health education at school is a possible intervention in LMICs.

Regarding socioeconomic factors, strengthening social welfare services for disadvantaged population and child maltreatment is recommended [56]. Allocating appropriate resources in this area in LMICs is an essential policy response. Delivering public education about child care are possible universal interventions in LMICs. 


\section{Limitations}

This study has some limitations. First, this study could only demonstrate the association of some socioeconomic and lifestyle factors with mental health. The cross-sectional design of this study precludes causal inferences. Second, as this study is a secondary analysis, this dataset was not optimized for the analysis of mental health risk factors. Therefore, some well-known risk factors such as maltreatment or abuse, parental mental health problems and perinatal complications were not adjusted [18]. Regarding basic socioeconomic factors, this study did not investigate the association of ethnicity, food security or community factors such as community deprivation and community safety [56]. Regarding lifestyle factors, this study did not investigate sedentary time or diet $[10,57]$.Third, this study excluded 607 participants with missing values for explanatory variables or the SDQ from the logistic regression analysis. The difference in socioeconomic background between participants without missing values and those with missing values may have caused a sampling bias. As more children from low socioeconomic groups were excluded from the analysis due to missing values, the association between socioeconomic variables and mental health problems might be underestimated. To address this issue, future studies should collect data through interviews to minimise the possibility of missing values, thereby reducing sampling bias.

Fourth, this study may have been affected by seasonal factors. The data were collected during the winter season, and it is possible that the season may also have affected participants' lifestyle. A severe winter might lead to further lifestyle changes, limiting physical activity and increasing screen time.

Lastly, this study was conducted in Ulaanbaatar, Mongolia's capital city. Children living in rural areas where more people are living as nomad were not included in this study. As the lifestyle of children living in the capital city and those in rural areas differ, lifestyle risk factors for rural children might also be different. For example, rural or nomadic children may exhibit higher levels of physical activity and spend less time on digital devices than do children in Ulaanbaatar, resulting in different risk levels for these factors. However, the results of the lifestyle factors might be applicable to children living in urban areas of LMICs, as less physical activity and longer screen time are common among children living in urban areas in LMICs. Thus, the findings of this study may be used as a basis to develop and implement health policies for improving the mental health of children and adolescents in Mongolia and similar countries.

\section{Conclusion}

Low socioeconomic status, especially low maternal education and short sleep, lack of physical activity, and long screen time were identified as factors associated with mental health problems among school-aged children in Mongolia. The results contribute to social implementation of preventive interventions in LMICs.

Supplementary Information The online version contains supplementary material available at https://doi.org/10.1007/s00127-021-02178-7.

Author contributions AA, KT, GT, and RM designed the study. GT, AT, NN, KZ, GL, and BS coordinated the logistics and supervised data collection and management. AA and KT performed the analysis. AA drafted the first manuscript. HS, AK, and KK critically reviewed the manuscript. All authors approved the final manuscript.

Funding This study was supported by JSPS KAKENHI, Grant number 17H04501 (to RM).

Data availability The datasets generated and/or analysed in the current study are not publicly available but are available from the corresponding author on reasonable request.

Code availability The statistical package that authors used in this analysis is publicly available.

\section{Declarations}

Conflict of interest The authors declare no conflicts of interest.

Ethical approval This study was approved by the ethical committee at National Center for Child Health and Development, Tokyo, Japan (Number 1541) and the Mongolian National Institute for Physical Education, Ulaanbaatar, Mongolia (Number 238).

Consent to participate Written consent to participate was obtained from the parents or guardians of the subjects.

Consent for publication Not applicable.

\section{References}

1. WHO. Child and adolescent mental health. https://www. who.int/mental_health/maternal-child/child_adolescent/en/. Accessed 25 June 2021

2. United Nations Department of Economic and Social Affairs. World Population Prospects 2019. New York: United Nations, 2019. https://population.un.org/wpp. Accessed 25 June 2021

3. Institute for Health Metrics and Evaluation (IHME) (2015) GBD Compare. http://vizhub.healthdata.org/gbd-compare. Accessed 25 June 2021

4. Kessler R, Amminger G, Aguilar-Gaxiola S, Alonso J, Lee S, Ustun T (2007) Age of onset of mental disorders: a review of recent literature. Curr Opin Psychiatry 20(4):359-364. https:// doi.org/10.1097/YCO.0b013e32816ebc8c 
5. Reiss F (2013) Socioeconomic inequalities and mental health problems in children and adolescents: a systematic review. Soc Sci Med 90:24-31. https://doi.org/10.1016/j.socscimed.2013. 04.026

6. Mittal VA, Firth J, Kimhy D (2020) Combating the dangers of sedentary activity on child and adolescent mental health during the time of COVID-19. J Am Acad Child Adolesc Psychiatry 59(11):1197-1198. https://doi.org/10.1016/j.jaac.2020.08.003

7. Moore SA, Faulkner G, Rhodes RE, Brussoni M, Chulak-Bozzer T, Ferguson LJ, Mitra R, O'Reilly N, Spence JC, Vanderloo LM, Tremblay MS (2020) Impact of the COVID-19 virus outbreak on movement and play behaviours of Canadian children and youth: a national survey. Int J Behav Nutr Phys Act 17(1):85. https://doi. org/10.1186/s12966-020-00987-8

8. Biddle SJH, Ciaccioni S, Thomas G, Vergeer I (2019) Physical activity and mental health in children and adolescents: an updated review of reviews and an analysis of causality. Psychol Sport Exerc 42:146-155. https://doi.org/10.1016/j.psychsport.2018. 08.011

9. Stiglic N, Viner RM (2019) Effects of screentime on the health and well-being of children and adolescents: a systematic review of reviews. BMJ Open 9(1):e023191. https://doi.org/10.1136/bmjop en-2018-023191

10. Hoare E, Milton K, Foster C, Allender S (2016) The associations between sedentary behaviour and mental health among adolescents: a systematic review. Int J Behav Nutr Phys Act 13(1):108. https://doi.org/10.1186/s12966-016-0432-4

11. Swing EL, Gentile DA, Anderson CA, Walsh DA (2010) Television and video game exposure and the development of attention problems. Pediatrics 126(2):214-221. https://doi.org/10.1542/ peds.2009-1508

12. Tamana SK, Ezeugwu V, Chikuma J, Lefebvre DL, Azad MB, Moraes TJ, Subbarao P, Becker AB, Turvey SE, Sears MR, Dick BD, Carson V, Rasmussen C, Cs I, Pei J, Mandhane PJ (2019) Screen-time is associated with inattention problems in preschoolers: results from the CHILD birth cohort study. PLoS ONE 14(4):e0213995. https://doi.org/10.1371/journal.pone.0213995

13. Marino C, Andrade B, Campisi SC, Wong M, Zhao H, Jing X, Aitken M, Bonato S, Haltigan J, Wang W, Szatmari P (2021) Association between disturbed sleep and depression in children and youths: a systematic review and meta-analysis of cohort studies. JAMA Netw Open 4(3):e212373. https://doi.org/10.1001/jaman etworkopen.2021.2373

14. Sampasa-Kanyinga H, Colman I, Goldfield GS, Janssen I, Wang J, Podinic I, Tremblay MS, Saunders TJ, Sampson M, Chaput JP (2020) Combinations of physical activity, sedentary time, and sleep duration and their associations with depressive symptoms and other mental health problems in children and adolescents: a systematic review. Int J Behav Nutr Phys Act 17(1):72. https://doi. org/10.1186/s12966-020-00976-x

15. WHO (2019) Guidelines on physical activity, sedentary behaviour and sleep for children under 5 years of age. World Health Organization. https://apps.who.int/iris/handle/10665/311664. Accessed 25 June 2021

16. Tremblay MS, Carson V, Chaput JP, Connor Gorber S, Dinh T, Duggan M, Faulkner G, Gray CE, Gruber R, Janson K, Janssen I, Katzmarzyk PT, Kho ME, Latimer-Cheung AE, LeBlanc C, Okely AD, Olds T, Pate RR, Phillips A, Poitras VJ, Rodenburg S, Sampson M, Saunders TJ, Stone JA, Stratton G, Weiss SK, Zehr L (2016) Canadian 24-hour movement guidelines for children and youth: an integration of physical activity, sedentary behaviour, and sleep. Appl Physiol Nutr Metab 41(6 Suppl 3):S311-327. https:// doi.org/10.1139/apnm-2016-0151

17. Department of Health, Australian Government (2021) Physical activity and exercise guidelines for all Australians For children and young people (5 to 17 years). https://www.health.gov.au/ health-topics/physical-activity-and-exercise/physical-activityand-exercise-guidelines-for-all-australians/for-children-andyoung-people-5-to-17-years. Accessed 25 June 2021

18. Kieling $\mathrm{C}$, Baker-Henningham $\mathrm{H}$, Belfer M, Conti G, Ertem I, Omigbodun O, Rohde LA, Srinath S, Ulkuer N, Rahman A (2011) Child and adolescent mental health worldwide: evidence for action. Lancet 378(9801):1515-1525. https://doi.org/10. 1016/s0140-6736(11)60827-1

19. Patel V, Saxena S, Lund C, Thornicroft G, Baingana F, Bolton P, Chisholm D, Collins PY, Cooper JL, Eaton J, Herrman H, Herzallah MM, Huang Y, Jordans MJD, Kleinman A, Medina-Mora ME, Morgan E, Niaz U, Omigbodun O, Prince M, Rahman A, Saraceno B, Sarkar BK, De Silva M, Singh I, Stein DJ, Sunkel C, UnÜtzer J (2018) The Lancet Commission on global mental health and sustainable development. Lancet 392(10157):15531598. https://doi.org/10.1016/s0140-6736(18)31612-x

20. World Health Organization and Calouste Gulbenkian Foundation (2014) Social determinants of mental health. World Health Organization. https://apps.who.int/iris/bitstream/handle/10665/ 112828/9789241506809_eng.pdf?sequence=1. Accessed 25 June 2021

21. Schady N (2011) Parents' education, mothers' vocabulary, and cognitive development in early childhood: longitudinal evidence from Ecuador. Am J Public Health 101(12):2299-2307. https:// doi.org/10.2105/AJPH.2011.300253

22. Murray J, Anselmi L, Gallo EA, Fleitlich-Bilyk B, Bordin IA (2013) Epidemiology of childhood conduct problems in Brazil: systematic review and meta-analysis. Soc Psychiatry Psychiatr Epidemiol 48(10):1527-1538. https://doi.org/10.1007/ s00127-013-0695-x

23. Samarakkody D, Fernando D, McClure R, Perera H, De Silva H (2012) Prevalence of externalizing behavior problems in Sri Lankan preschool children: birth, childhood, and sociodemographic risk factors. Soc Psychiatry Psychiatr Epidemiol 47(5):757-762. https://doi.org/10.1007/s00127-011-0377-5

24. Kinyanda E, Kizza R, Levin J, Ndyanabangi S, Abbo C (2011) Adolescent suicidality as seen in rural northeastern Uganda: prevalence and risk factors. Crisis 32(1):43-51. https://doi.org/ 10.1027/0227-5910/a000059

25. Perez-Marfil MN, Fernandez-Alcantara M, Fasfous AF, BurneoGarces C, Perez-Garcia M, Cruz-Quintana F (2020) Influence of socio-economic status on psychopathology in Ecuadorian children. Front Psychiatry 11:43. https://doi.org/10.3389/fpsyt.2020. 00043

26. Vancampfort D, Stubbs B, Firth J, Van Damme T, Koyanagi A (2018) Sedentary behavior and depressive symptoms among 67,077 adolescents aged 12-15 years from 30 low- and middleincome countries. Int J Behav Nutr Phys Act 15(1):73. https://doi. org/10.1186/s12966-018-0708-y

27. Wang MH, Xiao DM, Liu MW, Lu YA, He QQ (2020) Relationship between sedentary behaviour and anxiety symptoms among youth in 24 low- and middle-income countries. PLoS ONE 15(10):e0241303. https://doi.org/10.1371/journal.pone.0241303

28. Asare M, Danquah SA (2015) The relationship between physical activity, sedentary behaviour and mental health in Ghanaian adolescents. Child Adolesc Psychiatry Ment Health 9:11. https:// doi.org/10.1186/s13034-015-0043-x

29. Liu X (2004) Sleep and adolescent suicidal behavior. Sleep 27(7):1351-1358

30. Takehara K, Ganchimeg T, Kikuchi A, Gundegmaa L, Altantsetseg L, Aoki A, Fukuie T, Suwabe K, Bat-Erdene S, Mikami M, Mori R, Soya H (2019) The effectiveness of exercise intervention for academic achievement, cognitive function, and physical health among children in Mongolia: a cluster RCT study protocol. BMC Public Health 19(1):697. https://doi.org/10.1186/ s12889-019-6986-8 
31. Takehara K, Togoobaatar G, Kikuchi A, Gundegmaa L, Altantsetseg L, Aoki A, Fukuie T, Shagdar B, Suwabe K, Mikami M, Mori R, Soya H (2021) Exercise intervention for academic achievement among children: a randomized controlled trial. Pediatrics. https:// doi.org/10.1542/peds.2021-052808. Accepted 9 August 2021

32. World Bank (2019) The World Bank Open Data. https://data.world bank.org/. Accessed 25 June 2021

33. WHO (2014) World Health Organization-noncommunicable diseases (NCD) Country Profiles, 2014. https://apps.who.int/iris/ bitstream/handle/10665/128038/9789241507509_eng.pdf?seque nce $=1$. Accessed 25 June 2021

34. Davaasambuu S, Batbaatar S, Witte S, Hamid P, Oquendo MA, Kleinman M, Olivares M, Gould M (2017) Suicidal plans and attempts among adolescents in Mongolia. Crisis 38(5):330-343. https://doi.org/10.1027/0227-5910/a000447

35. Dagvadorj A, Corsi DJ, Sumya N, Muldoon K, Wen SW, Takehara K, Mori R, Walker MC (2019) Prevalence and determinants of mental health problems among children in Mongolia: a population-based birth cohort. Glob Epidemiol. https://doi.org/10.1016/j. gloepi.2019.100011

36. National Center for Public Health Mongolia (2013) Global School Based Student Health Survey Mongolia, 2013. Youth's Health Behaviour among Secondary School Children.

37. National Statistics Office of Mongolia (2019) Mongolian statistical information service. http://www.1212.mn/. Accessed 25 June 2021

38. Schelzig K, Newman K (2020) Promoting inclusive education in Mongolia. Asian Development Bank, Manila

39. youthinmind SDQinfo. https://www.sdqinfo.com/ Accessed 25 June 2021

40. youthinmind (2020) Downloadable SDQ and related items, Mongolian. One-sided SDQ for parents or teachers of 4-17 year olds. https://www.sdqinfo.org/py/sdqinfo/b3.py?language=Mongolian. Accessed 25 June 2021.

41. Goodman R (1997) The strengths and difficulties questionnaire: a research note. J Child Psychol Psychiatry 38(5):581-586. https:// doi.org/10.1111/j.1469-7610.1997.tb01545.x

42. Aoki A, Ganchimeg T, Naranbaatar N, Khishigsuren Z, Gundegmaa L, Bat-Erdene S, Munkhbaatar B, Mori R, Kikuchi A, Soya H, Kasai K, Takehara K (2021) Validation of the parent version of the strengths and difficulties questionnaire (SDQ) to screen mental health problems among school-age children in Mongolia. BMC Psychiatry 21(1):218. https://doi.org/10.1186/ s12888-021-03218-x

43. World Bank (2019) Mongolia's 2018 Poverty Rate Estimated at 28.4 Percent. https://www.worldbank.org/en/news/press-release/ 2019/06/21/mongolias-2018-poverty-rate-estimated-at-284-perce nt. Accessed 25 June 2021

44. WHO (2020) Physical activity. https://www.who.int/news-room/ fact-sheets/detail/physical-activity. Accessed 18 August 2021.

45. youthinmind (2016) Scoring the SDQ. https://www.sdqinfo.org/ py/sdqinfo/c0.py. Accessed 25 June 2021

46. Pelham WE, Foster EM, Robb JA (2007) The economic impact of attention-deficit/hyperactivity disorder in children and adolescents. Ambul Pediatr 7(1 Suppl):121-131. https://doi.org/10. 1016/j.ambp.2006.08.002
47. Erskine HE, Ferrari AJ, Nelson P, Polanczyk GV, Flaxman AD, Vos T, Whiteford HA, Scott JG (2013) Epidemiological modelling of attention-deficit/hyperactivity disorder and conduct disorder for the Global Burden of Disease Study 2010. J Child Psychol Psychiatry 54(12):1263-1274. https://doi.org/10.1111/jcpp.12144

48. Ferri SL, Abel T, Brodkin ES (2018) Sex differences in autism spectrum disorder: a review. Curr Psychiatry Rep 20(2):9. https:// doi.org/10.1007/s11920-018-0874-2

49. Gakidou E, Cowling K, Lozano R, Murray CJL (2010) Increased educational attainment and its effect on child mortality in 175 countries between 1970 and 2009: a systematic analysis. Lancet 376(9745):959-974. https://doi.org/10.1016/S0140-6736(10) 61257-3

50. Thomas MM, Gugusheff J, Baldwin HJ, Gale J, Boylan S, Mihrshahi S (2020) Healthy lifestyle behaviours are associated with children's psychological health: a cross-sectional study. Int J Environ Res Public Health. https://doi.org/10.3390/ijerph17207509

51. Robinson M, Kendall GE, Jacoby P, Hands B, Beilin LJ, Silburn SR, Zubrick SR, Oddy WH (2011) Lifestyle and demographic correlates of poor mental health in early adolescence. J Paediatr Child Health 47(1-2):54-61. https://doi.org/10.1111/j.1440-1754. 2010.01891.x

52. Özmert E, Toyran M, Yurdakök K (2002) Behavioral correlates of television viewing in primary school children evaluated by the child behavior checklist. Arch Pediatr Adolesc Med 156(9):910914. https://doi.org/10.1001/archpedi.156.9.910

53. Messing S, Rutten A, Abu-Omar K, Ungerer-Rohrich U, Goodwin L, Burlacu I, Gediga G (2019) How can physical activity be promoted among children and adolescents? A systematic review of reviews across settings. Front Public Health 7:55. https://doi. org/10.3389/fpubh.2019.00055

54. Ramsey Buchanan L, Rooks-Peck CR, Finnie RKC, Wethington HR, Jacob V, Fulton JE, Johnson DB, Kahwati LC, Pratt CA, Ramirez G, Mercer SL, Glanz K, Community Preventive Services Task F (2016) Reducing recreational sedentary screen time: a community guide systematic review. Am J Prev Med 50(3):402415. https://doi.org/10.1016/j.amepre.2015.09.030

55. Busch V, Altenburg TM, Harmsen IA, Chinapaw MJ (2017) Interventions that stimulate healthy sleep in school-aged children: a systematic literature review. Eur J Public Health 27(1):53-65. https://doi.org/10.1093/eurpub/ckw140

56. Lund C, Brooke-Sumner C, Baingana F, Baron EC, Breuer E, Chandra P, Haushofer J, Herrman H, Jordans M, Kieling C, Medina-Mora ME, Morgan E, Omigbodun O, Tol W, Patel V, Saxena S (2018) Social determinants of mental disorders and the sustainable development goals: a systematic review of reviews. Lancet Psychiatry 5(4):357-369. https://doi.org/10.1016/s22150366(18)30060-9

57. O'Neil A, Quirk SE, Housden S, Brennan SL, Williams LJ, Pasco JA, Berk M, Jacka FN (2014) Relationship between diet and mental health in children and adolescents: a systematic review. Am J Public Health 104(10):e31-e42. https://doi.org/10.2105/AJPH. 2014.302110 